\title{
Comorbid Diabetes and Severe Mental Illness: Outcomes in an Integrated Health Care Delivery System
}

\author{
Christina Mangurian, $M D, M A S^{1,2}$, Dean Schillinger, $M D^{2,3}$, John W. Newcomer, $M D^{4}$, \\ Eric Vittinghoff, $P h D^{5}$, Susan Essock, $P h D^{6}$, Zheng Zhu, $M S^{7}$, Wendy Dyer, $M S^{7}$, \\ Kelly C. Young-Wolff, $P h D, M P H^{1,7}$, and Julie Schmittdiel, $P h D^{7}$
}

\begin{abstract}
'Department of Psychiatry, Weill Institute of Neurosciences, University of California, San Francisco, San Francisco, CA, USA; ${ }^{2}$ UCSF Center for Vulnerable Populations at Zuckerberg San Francisco General Hospital, San Francisco, CA, USA; ${ }^{3}$ UCSF Division of General Internal Medicine at Zuckerberg San Francisco General Hospital, San Francisco, CA, USA; ${ }^{4}$ Thriving Mind South Florida and Washington University School of Medicine, St Louis, CA, USA; ${ }^{5}$ Department of Epidemiology and Biostatistics, University of California, San Francisco, San Francisco, CA, USA; ' $D e p a r t m e n t ~ o f$ Psychiatry, Columbia University, New York City, NY, USA; ${ }^{7}$ Kaiser Permanente Northern California Division of Research, Oakland, CA, USA.
\end{abstract}

\begin{abstract}
BACKGROUND: Diabetes prevalence is twice as high among people with severe mental illness (SMI) when compared to the general population. Despite high prevalence, care outcomes are not well understood.

OBJECTIVE: To compare diabetes health outcomes received by people with and without comorbid SMI, and to understand demographic factors associated with poor diabetes control among those with SMI.
\end{abstract}

DESIGN: Retrospective cohort study

PARTICIPANTS: 269,243 adults with diabetes

MAIN MEASURES: Primary outcomes included optimal glycemic control (Alc < 7) or poor diabetes control (Alc > 9) in 2014. Secondary outcomes included control of other cardiometabolic risk factors (hypertension, dyslipidemia, smoking) and recommended diabetes monitoring.

KEY RESULTS: Among this cohort, people with SMI ( $N=$ $4,399)$, compared to those without SMI $(N=264,844)$, were more likely to have optimal glycemic control, adjusting for various covariates (adjusted relative risk (aRR) $1.25,95 \%$ CI $1.21-1.28, p<.001)$ and less likely to have poor control (aRR 0.92, 95\% CI 0.87-0.98, $p=0.012$ ). Better blood pressure and lipid control was more prevalent among people with SMI when compared to those without SMI (aRR 1.03; 95\% CI 1.02-1.05, $p<.001$; aRR $1.02 ; 95 \%$ CI 1.00-1.05, $p=0.044$, respectively). No differences were observed in recommended Alc or LDL testing, but people with SMI were more likely to have blood pressure checked (aRR 1.02, 95\% CI 1.02-1.03, $p<.001$ ) and less likely to receive retinopathy screening (aRR 0.80, 95\% CI $0.71-0.91, p<.001)$ than those without SMI. Among people with diabetes and comorbid SMI, younger adults and Hispanics were more likely to have poor diabetes control.

CONCLUSIONS: Adults with diabetes and comorbid SMI had better cardiometabolic control than people with diabetes who did not have SMI, despite lower rates of retinopathy screening. Among those with comorbid SMI,

Electronic supplementary material The online version of this article (https://doi.org/10.1007/s11606-019-05489-3) contains supplementary material, which is available to authorized users.

Received October 30, 2018

Revised May 2, 2019

Accepted September 25, 2019

Published online November 8, 2019 younger adults and Hispanics were more vulnerable to poor Alc control.

KEY WORDS: diabetes; severe mental illness; healthcare delivery system; health outcomes.

J Gen Intern Med 35(1):160-6

DOI: $10.1007 / \mathrm{s} 11606-019-05489-3$

(C) Society of General Internal Medicine 2019

\section{INTRODUCTION}

US adults with severe mental illnesses (SMI) such as schizophrenia and bipolar disorder (numbering approximately 7 million Americans) die, on average, 25 years earlier than the general population, largely from premature cardiovascular disease (CVD). ${ }^{1}$ A recent large study of people with SMI found that $28 \%$ had diabetes, more than twice the prevalence of the general population during the same time frame. ${ }^{2}$ A prior systematic review of studies conducted between 1987 and 2005 also estimated a diabetes prevalence double that of age-matched samples from the general population during the same time period. ${ }^{3}$ Treatment with antipsychotic medications contributes to diabetes risk, with risk associated with both first and second-generation antipsychotics. ${ }^{4-6}$ Since diabetes is a potent risk-factor for $\mathrm{CVD},{ }^{7,8}$ it is important to examine treatment of diabetes among this vulnerable population.

Unfortunately, there have been no large, well-designed, recent studies evaluating diabetes care among diverse populations with SMI in the USA. Prior retrospective cohort studies found diabetes care to be comparable between patients with SMI and frequency matched controls, ${ }^{9}$ with good glucose control and medication adherence regardless of the location of their receipt of primary care. ${ }^{10}$ However, these studies were restricted to predominantly male samples in the Veterans Affairs Medical Center (VAMC), ${ }^{9-11}$ and a systematic review showed that diabetes care outcomes appear to be different for people with SMI dependent on whether examining VAMC or non-VAMC settings. ${ }^{12}$ In addition, Frayne et al. (2005) examined data that is over two decades old ${ }^{11}$ and was done prior to 
growing recognition of diabetes risk in this population associated with antipsychotic use, as evidenced by American Diabetes Association (ADA) guidelines and Healthcare Effectiveness Data and Information Set (HEDIS) measures. ${ }^{13,14}$

This study aimed to fill a gap in the literature by examining diabetes care outcomes received among a diverse population of adults with diabetes with and without SMI. We selected individuals with SMI who were treated with antipsychotic medications because of our prior work with this cohort, ${ }^{2,15}$ and also given the increased risk for metabolic side effects from these medications. ${ }^{4,16}$ We examined the correlates of poor diabetes control among the SMI cohort to identify subpopulations that may require more intensive interventions. To our knowledge, this is the first large study in over 20 years examining disparities in diabetes care among a racially and ethnically diverse populations with and without SMI.

\section{METHODS}

Study Design. This retrospective cohort study utilized electronic health record data from Kaiser Permanente Northern California's (KPNC). The study received approval by the UCSF Committee of Human Research and the Kaiser Permanente Northern California Institutional Review Board.

Setting. KPNC is an integrated delivery system serving over 4 million members in Northern California. KPNC provides inpatient, outpatient, pharmacy, and laboratory services, all linked through an integrated electronic health record, under capitated payment, and its membership is largely representative of the California population that it serves. People with SMI are typically cared for in KPNC specialty psychiatry departments that are integrated within the KPNC care delivery system with access to the full spectrum of outpatient, inpatient, laboratory, and pharmacy services. Psychiatric services are generally delivered within the same medical center complex as primary care services. Most patients with SMI receive psychotherapy and case management services, in addition to medication management with a psychiatrist. However, KPNC contracts out to county community mental health clinics for intensive case management, if necessary. The primary care providers are responsible for delivering diabetes care to these patients with SMI.

Population. The following inclusion criteria generated the cohort of patients with diabetes: (1) KPNC Diabetes registry, which includes people with any evidence of diabetes defined by a diabetes diagnosis (ICD-9 codes $250 . \mathrm{xx}$ and other medication parameters as previously described $)^{17}$ and (2) KPNC member with enrollment for at least 10 months in 2014. To define the SMI population, we included participants with a diagnosis of SMI as defined by at least one ICD-9 diagnosis (295.xx-301.x, 307.1, 307.5, 307.51, 309.81, 311.00, 314.xx, and 317.xx-329.xx) at any point during 2012-2014 and filled at least 2 prescriptions for an antipsychotic medication on different dates in 2014 (See Online Supplemental eTable 1 for list of medications). Our definition of SMI used all agreedupon diagnoses from two prior large studies (ICD-9 295.xx299.xx), ${ }^{18,19}$ and selected "other" diagnoses where psychiatrists commonly prescribe antipsychotic medications as described in other studies ${ }^{2}$ (See Online Supplemental eTable 2). We selected individuals treated with antipsychotic medications per our prior work with this cohort, ${ }^{2,15}$ and also given the increased risk for metabolic side effects from these medications. ${ }^{4,16}$ Individuals were excluded if they were younger than 18 years old.

Measures. The primary outcome measures were (1) good diabetes control, as measured by glycated hemoglobin (A1c) below $7 \%$, and (2) poor glucose control, as measured by A1c $>9 \%$ or no measurement that year. If there were two or more $\mathrm{A} 1 \mathrm{c}$ measures in the past year, we used the most recent A1c in that time period. Secondary outcome measures included control of other cardiovascular risk factors (blood pressure $<140 / 90$; LDL $<100$, current non-smoking status). In addition, to assess for processes of care and evidence of adherence to diabetes treatment guidelines, ${ }^{13}$ we examined additional outcomes including annual A1c test, annual blood pressure measurement, annual LDL laboratory test, and retinopathy screening for those with background diabetic retinopathy (ICD-9 362.01).

The database included additional variables: age, gender, race/ethnicity, geocoded census data (including urban area type, education, and median household income census variables), psychiatric diagnoses, antipsychotic medications, substance use disorder diagnoses (ICD-9 codes 303.xx through 305.xx), self-reported smoking status in 2013, body mass index (BMI) in 2013, presence or absence of CVD in 2013 (See Online Supplemental eTable 1), other CVD risk factors in 2013 (hypertension defined as ICD-9 codes 401.xx to 405.xx, Dyslipidemia defined by ICD-9 code 272.xx) and health care utilization during the index year (2014). If multiple psychiatric diagnoses were documented, an individual was categorized based on a hierarchy with priority order as follows: schizophrenia and other psychotic disorders, affective disorders, autism, and other. If an individual had multiple health insurance types, they were categorized based on a hierarchy with priority order as follows: (1) Medicaid, (2) Medicare, (3) commercial, and (4) other. So, for example, if anyone had both Medicare and commercial insurance, the insurance type for the patient would be Medicare. We chose these demographic, psychiatric, and medical variables to characterize the sample and for use as covariates, since some are associated with glycemic control as described below.

Assessment for Missing Data. To determine the completeness of our data and ensure that laboratory information was not 
occurring outside of KPNC, we searched claims data for evidence of diabetes screening labs (CPT codes 82947, 82950, 82951, 83036) drawn outside of the KPNC system. We found that less than $0.4 \%$ of laboratory tests were performed outside of KPNC.

Statistical Analysis. We used chi-square tests to evaluate differences in demographic, insurance, and clinical characteristics and diabetes care outcomes between patients with SMI vs. without SMI. We then used Poisson models ${ }^{20}$ with robust standard errors to estimate the associations of SMI with diabetes control and care outcomes, adjusting for smoking status, age, gender, race, urban area type, BMI, primary care utilization, and medical facility. Since glycemic control are often personalized based on co-morbidity and some suggest a cutoff of $<8 \%,{ }^{21}$ we also conducted a sensitivity analysis in which the threshold for A1c control was defined as A1c $<8 \%$. In addition, we used a Poisson model to examine whether age, gender, race/ethnicity, comorbid substance use, BMI, or primary care utilization - all variables that could impact glycemic control —were independently associated with poor control among people with SMI. Finally, we compared the adjusted means of A1c, LDL, and blood pressure levels between patients with SMI vs. without SMI. For these models, we used a directed acyclic graph (DAG) to identify confounders and mediators of each predictor of poor diabetes care as described previously. ${ }^{15}$ We used the confounders as covariates in our analysis.

\section{RESULTS}

Applying our inclusion and exclusion criteria, we identified a large cohort of people with diabetes $(N=269,243)$. The demographics of this population are described in Table 1. At baseline, there were significant differences in demographic and clinical characteristics and utilization of health care (Table 1). For example, there were more women $(60 \%$ vs $47 \%, p<0.001$ ), more whites ( $58 \%$ vs $44 \%, p<.0001)$, more insured by Medicaid ( $10 \%$ vs $2 \%, p<.0001)$, higher mean BMI (36.6 vs 31.4, $p<.0001$ ), higher rates of comorbid disorders, and higher median health care utilization in the group with SMI compared to those without SMI. Among those with SMI, the vast majority $(70 \%)$ of antipsychotic prescriptions were for 30 or 100 days.

Among people with diabetes, those with comorbid SMI were more likely to have optimal diabetes control than those without SMI ( $51 \%$ vs $40 \%, p<0.0001$, Table 2 ).

After controlling for age, gender, race, urban area type, BMI, primary care utilization and medical facility, people with SMI were $25 \%$ more likely to have good diabetes control (adjusted relative risk $[\mathrm{aRR}]=1.25,95 \%$ CI 1.21-1.28, $p<$ $.001)$ compared to those without SMI (Table 3). With optimal diabetes control was defined as A1c $<8 \%$, those with SMI continued to have better control (aRR 1.06, 95\% CI 1.04$1.08, \mathrm{p}<0.001)$. The mean adjusted A1c was lower in people with SMI (7.16, 95\% CI 7.11-7.21) compared to people without SMI (7.47; 95\% CI 7.46-7.47, $p<0.001)$. In addition, people with SMI were less likely to have poor diabetes control (aRR 0.92, 95\% CI 0.87-0.98, $p<.012$ ). People with SMI were more likely to have well-controlled blood pressure (RR $1.03,95 \%$ CI $1.02-1.05, p<0.001)$ and mean adjusted systolic blood pressure (SBP) and diastolic blood pressure (DBP) was lower in people with SMI (SBP: $125 \mathrm{mmHg}$, 95\% CI 124.7-125.6; DBP 71.1, 95\% CI 70.8-71.5) compared to people without SMI (SBP: $128 \mathrm{mmHg}$, 95\% CI 127.9128.1; DBP 71.6, 95\% CI 71.5-71.6, $p<0.001$ ). People with SMI were more likely to have well-controlled lipids (RR 1.02, 95\% CI 1.00-1.05, $p=0.044$ ), and a lower mean adjusted LDL (85; 95\% CI 84.3-86.4) compared to people without SMI (88; 95\% CI 88.2-88.5, $p<0.001)$. People with SMI were less likely to have quit smoking (aRR 0.94, 95\% CI $0.93-0.96, p=.001$ ). Finally, people with SMI were slightly more likely to have recommended blood pressure measurements (aRR 1.02, 95\% CI 1.02-1.03, $p<.001$ ) but substantially less likely to have retinopathy screening (aRR 0.80,95\% CI $0.71,0.91, p<.001$ ), while A1c and LDL testing rates were similar between groups.

Among the cohort of people with SMI and diabetes, our adjusted analysis found that young adults, Hispanics, and those without any primary care visits were more likely to have poor diabetes control (see Fig. 1). Specifically, young adults ${ }^{18-}$ ${ }^{29}$ had over twice the risk of having poor control compared to older adults (aRR 2.15, 95\% CI 1.65-2.79, $p<0.001$ ), Hispanics were $18 \%$ more likely to have poor control compared to whites (aRR 1.18, 95\% CI 1.01-1.39, $p$ value 0.041 ), and people without any primary care visits were over twice as likely to have poor control when compared to those who frequently utilized primary care services (aRR 2.72 , 95\% CI $2.23-3.33, p<0.001)$.

\section{DISCUSSION}

In this cohort of people with diabetes in an integrated health care delivery system, we found that those with SMI had better glycemic control than people with who did not have SMI. We also found better control of hypertension and dyslipidemia, no difference in A1c or LDL lab monitoring, but improved screening for blood pressure. All of these findings indicate that people with diabetes and comorbid SMI appeared to have better diabetes health outcomes and process of care outcomes than those without SMI.

Our findings are particularly noteworthy because people with SMI are increasingly identified as a health disparities population because of their high mortality rates and frequently low-quality care. ${ }^{22}$ For example, in Medicaid populations served in the fragmented public health care system, studies consistently show that only $30 \%$ of people with SMI are 
Table 1 Characteristics of People in the Diabetes Registry With and Without SMI

\begin{tabular}{|c|c|c|c|}
\hline Characteristic & $\begin{array}{l}\text { Diabetes with SMI* } \\
(N=4,399)\end{array}$ & $\begin{array}{l}\text { Diabetes without } \mathrm{SMI} \dagger \\
(N=\mathbf{2 6 4 , 8 4 4 )}\end{array}$ & $P$ value \\
\hline \multicolumn{4}{|l|}{ Demographic characteristics } \\
\hline Female gender & $60 \%$ & $47 \%$ & $<.0001$ \\
\hline Race/ethnicity & & & $<.0001$ \\
\hline African American & $11 \%$ & $10 \%$ & \\
\hline Asian & $10 \%$ & $21 \%$ & \\
\hline Hispanic & $18 \%$ & $21 \%$ & \\
\hline White & $58 \%$ & $44 \%$ & \\
\hline Other & $1 \%$ & $2 \%$ & \\
\hline Unknown & $2 \%$ & $3 \%$ & \\
\hline \multicolumn{4}{|l|}{ Age, years } \\
\hline Mean (SD) & $60.5(15.0)$ & $61.9(13.6)$ & $<.0001$ \\
\hline Minimum, maximum & 18,98 & 18,106 & \\
\hline Age categories & & & $<.0001$ \\
\hline $18-29$ & $2 \%$ & $1 \%$ & \\
\hline $30-39$ & $6 \%$ & $4 \%$ & \\
\hline $40-49$ & $14 \%$ & $12 \%$ & \\
\hline $50-59$ & $26 \%$ & $24 \%$ & \\
\hline $60-64$ & $13 \%$ & $14 \%$ & \\
\hline $65+$ & $38 \%$ & $44 \%$ & \\
\hline Insurance (hierarchy) $\ddagger$ & & & $<.0001$ \\
\hline Medicaid & $10 \%$ & $2 \%$ & \\
\hline Medicare & $62 \%$ & $47 \%$ & \\
\hline Commercial & $28 \%$ & $51 \%$ & \\
\hline Other & $<1 \%$ & $<1 \%$ & \\
\hline \multicolumn{4}{|l|}{ Clinical characteristics $\S$} \\
\hline \multicolumn{4}{|l|}{ BMI } \\
\hline Mean (SD) & $36.6(7.9)$ & $31.4(7.1)$ & $<.0001$ \\
\hline Minimum, maximum & 15,87 & 15,89 & \\
\hline BMI categories & & & $<.0001$ \\
\hline Normal $(<25$ kg/m²) & $14 \%$ & $14 \%$ & \\
\hline Overweight $\left(25-<30 \mathrm{~kg} / \mathrm{m}^{2}\right)$ & $23 \%$ & $28 \%$ & \\
\hline Obese $\left(\geq 30 \mathrm{~kg} / \mathrm{m}^{2}\right)$ & $53 \%$ & $45 \%$ & \\
\hline Unknown & $10 \%$ & $13 \%$ & \\
\hline Prior smoker & $56 \%$ & $44 \%$ & $<.0001$ \\
\hline Substance use disorders\| & $21 \%$ & $6 \%$ & $<.0001$ \\
\hline Hypertension\| & $69 \%$ & $65 \%$ & $<.0001$ \\
\hline Dyslipidemiall & $77 \%$ & $69 \%$ & $<.0001$ \\
\hline CVD $\|$ & $28 \%$ & $21 \%$ & $<.0001$ \\
\hline \multicolumn{4}{|l|}{ Health care utilization characteristics } \\
\hline \multicolumn{3}{|l|}{ Number of primary care visits] } & $<.0001$ \\
\hline 0 & $7 \%$ & $12 \%$ & \\
\hline $1-2$ & $29 \%$ & $39 \%$ & \\
\hline $3-4$ & $24 \%$ & $24 \%$ & \\
\hline $5+$ & $39 \%$ & $26 \%$ & \\
\hline (Among 5+): min, median, $\max$ & $\operatorname{Min}=5, \operatorname{Med}=8, \operatorname{Max}=130$ & $\operatorname{Min}=5, \operatorname{Med}=7, \operatorname{Max}=156$ & \\
\hline \multicolumn{3}{|l|}{ Number of mental health visits] } & $<.0001$ \\
\hline 0 & $38 \%$ & $95 \%$ & \\
\hline $1-2$ & $29 \%$ & $3 \%$ & \\
\hline $3-4$ & $10 \%$ & $1 \%$ & \\
\hline $5+$ & $23 \%$ & $1 \%$ & \\
\hline (Among 5+): min, median, $\max$ & $\operatorname{Min}=5, \operatorname{Med}=11, \operatorname{Max}=119$ & $\operatorname{Min}=5, \operatorname{Med}=8, \operatorname{Max}=97$ & \\
\hline \multicolumn{3}{|c|}{ Total number of primary care and mental health visits II } & $<.0001$ \\
\hline 0 & $4 \%$ & $11 \%$ & \\
\hline $1-2$ & $17 \%$ & $38 \%$ & \\
\hline $3-4$ & $18 \%$ & $24 \%$ & \\
\hline $5+$ & $60 \%$ & $27 \%$ & \\
\hline (Among 5+): min, median, $\max$ & $\operatorname{Min}=5, \operatorname{Med}=10, \operatorname{Max}=141$ & $\operatorname{Min}=5, \operatorname{Med}=7, \operatorname{Max}=156$ & \\
\hline
\end{tabular}

*Defined as meeting criteria for SMI per Online Supplemental eTable 2 and in the KPNC DOR diabetes registry as described below + Control group consists of patients in the KPNC DOR diabetes registry as of 12/31/2014 excluding patients who were SMI, $<18$ years old, unknown gender, enrolled less than 10 months in 2014, or died prior to 2015

If an individual had multiple health insurance types, they were categorized based on a hierarchy with the following priority order: (1) Medicaid, (2) Medicare, (3) commercial, (4) other

\$Based on last measurement in 2013

||Substance abuse diagnosis includes any of the following ICD-9 codes: 303.x to 305.xHypertension diagnosis includes any of the following ICD-9 codes: 401.x to 405.xDyslipidemia diagnosis includes any of the following ICD-9 codes: 272.xCVD diagnosis includes any of the following ICD-9 codes: 402.x, 404.x, 410.x, 412.x to 414.x, 427.x to 431.x, 433.x to 434.x, 436.x.437.0, 437.1, 437.2, 437.8, 437.9

IA maximum of one visit per day was counted within each visit category

screened for diabetes, ${ }^{23-25}$ though our recent work in KPNC found higher screening rates $(55 \%){ }^{15}$ That increased diabetes screening is critical because of the burden of disease, with $28 \%$ of those screened having diabetes and $47 \%$ having 
Table 2 Diabetes Outcomes and Processes of Care Among Adults in the KP Diabetes Registry With and Without SMI, Unadjusted

\begin{tabular}{|c|c|c|c|}
\hline $\begin{array}{l}\text { Diabetes care } \\
\text { outcomes* }\end{array}$ & $\begin{array}{l}\text { Diabetes with } \\
\text { SMI } \dagger \\
(N=4,399)\end{array}$ & $\begin{array}{l}\text { Diabetes without } \\
\text { SMI }+ \\
(N=\mathbf{2 6 4 , 8 4 4 )}\end{array}$ & $P$ value \\
\hline \multicolumn{4}{|l|}{ Primary outcome } \\
\hline $\begin{array}{l}\text { Optimal diabetes } \\
\text { control } \\
(\mathrm{A} 1 \mathrm{c}<7 \%) \S\end{array}$ & $2,232(51 \%)$ & $107,043(40 \%)$ & $<.0001$ \\
\hline $\begin{array}{l}\text { Poor diabetes } \\
\text { control } \\
(\text { Alc }>9) \| \\
\text { Secondary outcomes }\end{array}$ & $816(19 \%)$ & $51,687(20 \%)$ & 0.1086 \\
\hline \multicolumn{4}{|c|}{ Control of other cardiovascular risk factors } \\
\hline $\mathrm{BP}<140 / 90 \S$ & $3,683(84 \%)$ & $215,710(81 \%)$ & 0.0001 \\
\hline LDL $<100 \S$ & $2,678(61 \%)$ & $162,608(61 \%)$ & 0.4822 \\
\hline $\begin{array}{l}\text { Current } \\
\text { non-smokerd }\end{array}$ & $3,428(78 \%)$ & $210,016(79 \%)$ & 0.0261 \\
\hline \multicolumn{4}{|c|}{$\begin{array}{l}\text { non-smoker } \| \\
\text { Recommended diabetes monitoring }\end{array}$} \\
\hline $\begin{array}{l}\text { Annual A1c } \\
\text { lab test }\end{array}$ & $4,048(92 \%)$ & $243,358(92 \%)$ & 0.7475 \\
\hline $\begin{array}{l}\text { Blood pressure } \\
\text { measurement }\end{array}$ & $4,225(96 \%)$ & $246,849(93 \%)$ & $<.0001$ \\
\hline LDL lab test & $3,763(86 \%)$ & $228,708(86 \%)$ & 0.1191 \\
\hline $\begin{array}{l}\text { Retinopathy } \\
\text { screening \# }\end{array}$ & $122(52 \%)$ & $7,385(66 \%)$ & $<.0001$ \\
\hline
\end{tabular}

*All measurements took place in 2014

tDefined as meeting criteria for SMI per Online Supplemental eTable 2 and in the KPNC DOR diabetes registry as described below

+ Control group consists of patients in the KPNC DOR diabetes registry as of 12/31/2014 excluding patients who were SMI, < 18 years old, unknown gender, enrolled less than 10 months in 2014, or died prior to 2015

\$Based on last measurement in 2014

$\|$ Alc $>9 \%$ or no measurement

- Smoking status defined as current smoker or unknown in 2013

\#Sample restricted to patients with background retinopathy in 2013 (N $=11,500)$. Diabetes with $S M I(n=233)$ and diabetes without $\operatorname{SMI}(N=$ $11,267)$

prediabetes..$^{2}$ In addition, from a population viewpoint, even small reductions in blood pressure can have very meaningful impacts upon all populations in terms of risk of myocardial infarction and stroke. ${ }^{26}$ These blood pressure reductions are especially important for those who at high risk for cardiovascular disease, like people with diabetes and people living with serious mental illness. We believe future comparativeeffectiveness trials should be done to understand what components of this delivery system actually drive the reduction in disparities in care for this vulnerable population.

In addition, we found disparities in diabetes control among subpopulations with SMI, specifically young adults and Hispanics, which is consistent with prior work in the general population. ${ }^{27,28}$ This adds to the growing evidence that young adults and minorities with SMI could benefit from enhanced screening and early identification of diabetes. ${ }^{2,15,16,23}$

It is possible that these positive health care outcomes may be a direct result of better adherence to diabetes medications. In prior studies, people with mental illness had more selfreported adherence to diabetes medications. ${ }^{29-31}$ Authors have speculated that managing a chronic mental illness might confer advantages to people with SMI in the self-care of other conditions like diabetes, or that these patients have additional members of the health care team (psychiatrists, case managers)
Table 3 Diabetes Care Outcomes Among People With and Without SMI, Adjusted

\begin{tabular}{|c|c|c|}
\hline $\begin{array}{l}\text { Dependent variable } \\
\text { (outcome)* }\end{array}$ & $\begin{array}{l}\text { Adjusted RR for SMI } \\
\text { with } \mathrm{CI} \dagger\end{array}$ & $\begin{array}{l}P \\
\text { value }\end{array}$ \\
\hline \multicolumn{3}{|l|}{ Primary outcomes } \\
\hline $\begin{array}{l}\text { Optimal diabetes control (A1c } \\
<7 \%) \$\end{array}$ & $1.25(1.21,1.28)$ & $<.001$ \\
\hline $\begin{array}{l}\text { Poor diabetes control (A1c > } \\
\text { ( }\end{array}$ & $0.92(0.87,0.98)$ & $<.012$ \\
\hline \multicolumn{3}{|l|}{ Secondary outcomes } \\
\hline \multicolumn{3}{|c|}{ Control of other cardiovascular risk factors } \\
\hline $\mathrm{BP}<140 / 90 \ddagger$ & $1.03(1.02,1.05)$ & $<.001$ \\
\hline LDL $<100 \ddagger$ & $1.02(1.00,1.05)$ & 0.044 \\
\hline Current non-smoker\| & $0.94(0.93,0.96)$ & 0.001 \\
\hline \multicolumn{3}{|c|}{ Recommended diabetes monitoring } \\
\hline $\begin{array}{l}\text { Annual A1c lab test } \\
\text { completed }\end{array}$ & $1.00(0.99,1.01)$ & 0.834 \\
\hline Blood pressure measurement & $1.02(1.02,1.03)$ & $<.001$ \\
\hline LDL lab test & $0.99(0.98,1.01)$ & 0.323 \\
\hline Retinopathy screening & $0.80(0.71,0.91)$ & 0.001 \\
\hline
\end{tabular}

*All measurements took place in 2014

Estimates from modified Poisson regressions with robust standard errors, using SMI (vs. no SMI) as the main independent variable, and controlling for age, gender, race, urban area type, BMI, primary care utilization, and medical facility. Number of observations $=269,243$

tBased on last measurement in 2014

$\S A 1 \mathrm{l}>9 \%$ or no measurement

IISmoking status defined by as current smoker or unknown in 2013

- Sample restricted to patients with background retinopathy in 2013 (N $=11,959)$. Diabetes with SMI $(n=235)$ and diabetes without $\operatorname{SMI}(N=$ $11,724)$

and early and sustained involvement in the health system at large.

Although this is a rigorous and large study, the major limitation of this study may be generalizability, since the data come from one delivery system, albeit a very large one. In addition, there is an overrepresentation of Caucasians in this sample (68\%) when compared with the general KPNC population ( $43 \%$ Caucasian). Although this difference is consistent with prior studies of KPNC member's mental health utilization, ${ }^{32}$ it may influence generalizability of findings to more diverse populations and uninsured or publicly insured populations. The study is also limited by lack of data on longitudinal exposure to antipsychotic medications that are risk factors for diabetes. In addition, there was significant missingness for the current smoking variable, with an "unknown" status for $8 \%$ of the SMI population and $14 \%$ of the non-SMI population, so the rates of non-smoking might be higher in both groups.

This work has major public health implications. Given the excellent diabetes care outcomes obtained for this population with severe mental illness receiving care in a system that champions population health approaches, it is worth considering whether such approaches might reduce disparities for this vulnerable population in other settings. Future comparative-effectiveness trials should be done to understand what specific components of this delivery system helped to mitigate health care disparities for this vulnerable population. Future research should also examine quality of prediabetes care among this population compared to the general population. 


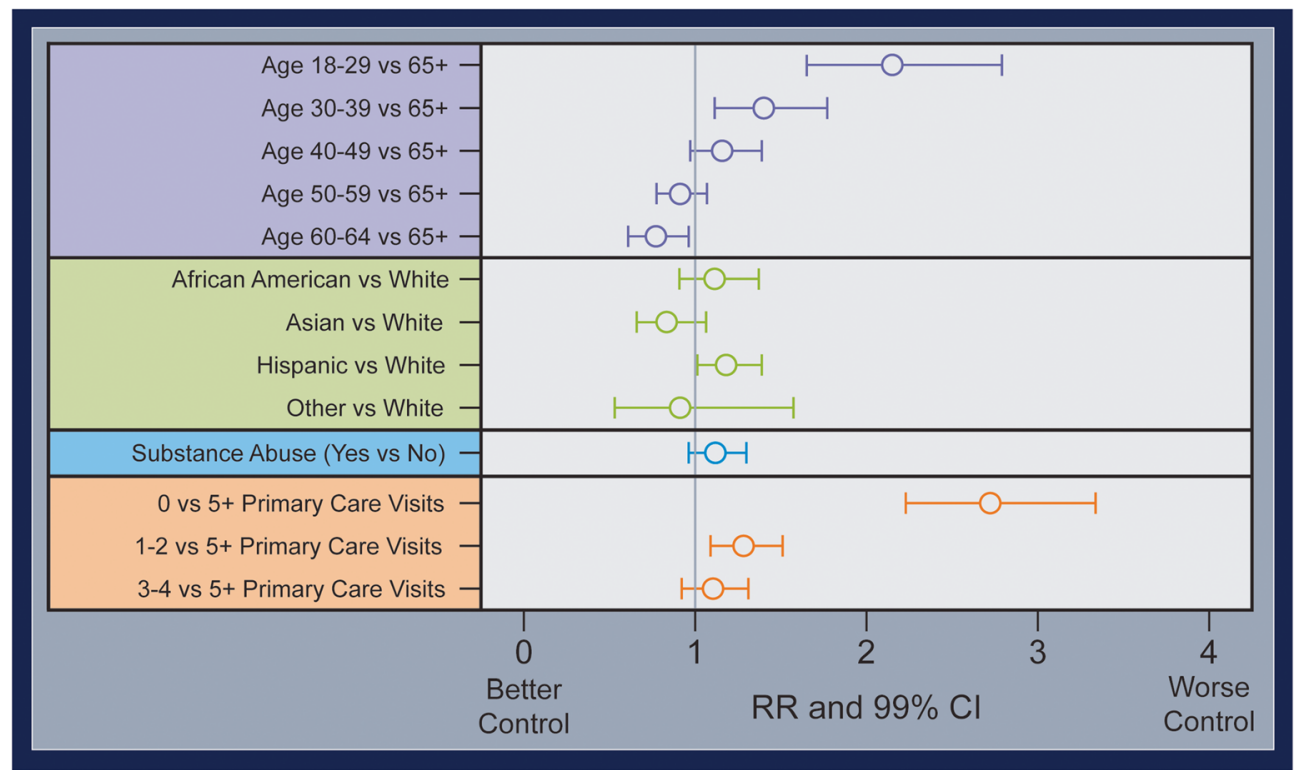

Figure 1 Potential factors associated with poor diabetes control among people with SMI $(N=4,339)$. Out of 4,399 individuals with SMI and DM, 816 had at least one A1c $>9$ or no A1c measurement in 2014. Effect estimates are adjusted for confounders but not mediators: age is adjusted for sex, race/ethnicity, and medical facility; race/ethnicity for age, sex, and medical facility; substance abuse for age, sex, race/ethnicity, county, education, psychiatric diagnosis, past mental health utilization, past primary care utilization and medical facility; and primary care utilization for urban area type, education, income, insurance, psychiatric diagnosis, psychotropic adherence, substance Abuse, medical facility, cardiovascular disease, current primary care utilization, current mental health utilization, past mental health utilization, past primary care utilization and medical facility.

\section{CONCLUSION}

This is the first large, comprehensive study to examine current diabetes care in a diverse sample of adults with diabetes and comorbid SMI in the USA. In this large cohort, we found that people with diabetes and comorbid SMI had better control of three major cardiovascular risk factors (diabetes, hypertension, and dyslipidemia) than people with diabetes who did not have SMI. We also found room for improvement in smoking cessation and retinopathy screening. Finally, we found that among people with SMI and co-morbid diabetes young adults, and those with Hispanic ethnicity were more likely to have poor control. Future research should be done to understand which components of care delivery are most helpful in reversing health care disparities for this vulnerable population.

Acknowledgments: Thanks to UCSF Assistant Clinical Research Coordinator Nicholas S. Riano, MAS, for his assistance in preparing the manuscript. Thanks to Dr. Constance Weisner for her scientific consultation on our findings and KPNC Psychiatry leadership (Drs. Don Mordecai and Mason Turner) for their thoughtful input on the draft.

Corresponding Author: Christina Mangurian, MD, MAS; Department of Psychiatry, Weill Institute of Neurosciences University of California, San Francisco, San Francisco, CA, USA (e-mail: christina. mangurian@ucsf.edu).

Funding Information All authors received support from a grant from the NIH National Institute of Diabetes and Digestive and Kidney Diseases (NIDDK) (RO3 DK101857). Drs. Schillinger and Schmittdiel received support from the Health Delivery Systems Center for Diabetes Translational Research (CDTR) (NIDDK P30 DK092924).

\section{Compliance with Ethical Standards:}

The study received approval by the UCSF Committee of Human Research and the Kaiser Permanente Northern California Institutional Review Board.

Conflict of Interest: Dr. Mangurian was supported by an $\mathrm{NIH}$ Career Development Award (K23MH093689). Dr. Newcomer has grant support from Otsuka America Pharmaceutical Inc., consulting fees from Sunovion Pharmaceuticals, and he serves on a Data Safety Monitoring Board for Amgen, outside the submitted work. Dr. Schillinger received support from NIH Center grant P60MD006902. Dr. Essock received consulting income from the National Association of State Mental Health Program Directors and RAND Corporation. All remaining authors declare that they do not have a conflict of interest.

\section{REFERENCES}

1. Colton CW, Manderscheid RW. Congruencies in increased mortality rates, years of potential life lost, and causes of death among public mental health clients in eight states. Prev Chronic Dis 2006;3(2):A42.

2. Mangurian CV, Schillinger D, Newcomer JW, Vittinghoff E, Essock SM, Zhu Z, et al. Diabetes and Prediabetes Prevalence by Race and Ethnicity Among People With Severe Mental Illness. Diabetes Care 2018. https://doi.org/10.2337/dc18-0425

3. Osborn DP, Wright CA, Levy G, King MB, Deo R, Nazareth I. Relative risk of diabetes, dyslipidaemia, hypertension and the metabolic syndrome in people with severe mental illnesses: systematic review and metaanalysis. BMC Psychiatry 2008;8:84. https://doi.org/10.1186/ $1471-244 \mathrm{x}-8-84$ 
4. Newcomer JW. Second-generation (atypical) antipsychotics and metabolic effects: a comprehensive literature review. CNS Drugs 2005;19(Suppl 1):1-93.

5. Mangurian C, Newcomer JW, Modlin C, Schillinger D. Diabetes and Cardiovascular Care Among People with Severe Mental Illness: A Literature Review. J Gen Intern Med 2016:1-9. https://doi.org/10. 1007/s11606-016-3712-4

6. Rajkumar AP, Horsdal HT, Wimberley T, Cohen D, Mors O, Børglum AD, et al. Endogenous and Antipsychotic-Related Risks for Diabetes Mellitus in Young People With Schizophrenia: A Danish Population-Based Cohort Study. Am J Psychiatr 2017;174(7):686-94.

7. Kreyenbuhl J, Dickerson FB, Medoff DR, Brown CH, Goldberg RW, Fang $\mathbf{L}$, et al. Extent and management of cardiovascular risk factors in patients with type 2 diabetes and serious mental illness. J Nerv Ment Dis 2006;194(6):404-10. https://doi.org/10.1097/01.nmd.0000221177. $51089.7 \mathrm{~d}$

8. Ford ES. Risks for all-cause mortality, cardiovascular disease, and diabetes associated with the metabolic syndrome: a summary of the evidence. Diabetes Care 2005;28(7):1769-78.

9. Krein SL, Bingham CR, McCarthy JF, Mitchinson A, Payes J, Valenstein M. Diabetes treatment among VA patients with comorbid serious mental illness. Psychiatr Serv 2006;57(7):1016-21. https://doi. org/10.1176/appi.ps.57.7.1016

10. Long JA, Wang A, Medvedeva EL, Eisen SV, Gordon AJ, Kreyenbuhl J, et al. Glucose control and medication adherence among veterans with diabetes and serious mental illness: does collocation of primary care and mental health care matter? Diabetes Care 2014;37(8):2261-7.

11. Frayne SM, Halanych JH, Miller DR, Wang F, Lin H, Pogach L, et al. Disparities in diabetes care: impact of mental illness. Arch Intern Med 2005;165(22):2631-8. https://doi.org/10.1001/archinte.165.22.2631

12. Gierisch JM, Beadles C, Shapiro A, McDuffie J, Cunningham N, Bradford D, et al. Health disparities in quality indicators of healthcare among adults with mental illness. 2014.

13. National Committee for Quality Assurance. HEDIS Measures. 2017. http://www.ncqa.org/hedis-quality-measurement/hedis-measures.

14. American Diabetes Association. Standards of medical care in diabetes2018. Diabetes Care. 2018;41(Supplement 1):S1-S159.

15. Mangurian $\mathbf{C}$, Schillinger $\mathbf{D}$, Newcomer $\mathbf{J W}$, Vittinghoff $\mathbf{E}$, Essock $\mathbf{S}$, Zhu Z, et al. Diabetes Screening among Antipsychotic-Treated Adults with Severe Mental Illness in an Integrated Delivery System: A Retrospective Cohort Study. J Gen Intern Med 2017. https://doi.org/10. 1007/s11606-017-4205-9

16. Mangurian C, Newcomer JW, Modlin C, Schillinger D. Diabetes and Cardiovascular Care Among People with Severe Mental Illness: A Literature Review. J Gen Intern Med 2016;31(9):1083-91. https://doi. org/10.1007/s11606-016-3712-4

17. Schmittdiel JA, Uratsu CS, Fireman BH, Selby JV. The effectiveness of diabetes care management in managed care. Am J Manag Care 2009; 15(5):295-301

18. Simoni-Wastila L, Zuckerman IH, Shaffer T, Blanchette CM, Stuart B Drug use patterns in severely mentally ill Medicare beneficiaries: impact of discontinuities in drug coverage. Health Serv Res 2008;43(2):496-514
19. Druss BG, Bradford DW, Rosenheck RA, Radford MJ, Krumholz HM. Mental disorders and use of cardiovascular procedures after myocardial infarction. Jama. 2000;283(4):506-11.

20. Zou G. A modified poisson regression approach to prospective studies with binary data. Am J Epidemiol 2004;159(7):702-6.

21. Morales J, Assumpcao-Morales M. The 2018 update of the American College of Physicians glycaemic management recommendations: An invitation to continued inertia? Diabetes, Obes Metab 2018;20(8):1809-11.

22. Goldman ML, Spaeth-Rublee B, Pincus HA. The case for severe mental illness as a disparities category. Psychiatric services. 2018:appi. ps. 201700138.

23. Mangurian C, Newcomer JW, Vittinghoff E, Creasman JM, Knapp P, Fuentes-Afflick E, et al. Diabetes Screening Among Underserved Adults With Severe Mental Illness Who Take Antipsychotic Medications. JAMA Intern Med 2015;175(12):1977-9. https://doi.org/10.1001/jamainternmed.2015.6098

24. Morrato EH, Druss B, Hartung DM, Valuck RJ, Allen R, Campagna E, et al. Metabolic testing rates in 3 state Medicaid programs after FDA warnings and ADA/APA recommendations for second-generation antipsychotic drugs. Arch Gen Psychiatry 2010;67(1):17-24. https://doi.org/ 10.1001/archgenpsychiatry.2009.179

25. Essock SM, Covell NH, Leckman-Westin E, Lieberman JA, Sederer LI, Kealey E, et al. Identifying clinically questionable psychotropic prescribing practices for medicaid recipients in new york state. Psychiatr Serv 2009;60(12):1595-602. https://doi.org/10.1176/appi.ps.60.12.1595

26. Bibbins-Domingo K, Chertow GM, Coxson PG, Moran A, Lightwood JM, Pletcher MJ, et al. Projected effect of dietary salt reductions on future cardiovascular disease. N Engl J Med 2010;362(7):590-9.

27. Fernandez A, Schillinger D, Warton EM, Adler N, Moffet HH, Schenker Y, et al. Language barriers, physician-patient language concordance, and glycemic control among insured Latinos with diabetes: the Diabetes Study of Northern California (DISTANCE). J Gen Intern Med 2011;26(2):170-6.

28. Kunasegaran S, Beig J, Khanolkar M, Cundy T. Adherence to medication, glycaemic control and hospital attendance in young adults with type 2 diabetes. Intern Med J 2018;48(6):728-31.

29. Kreyenbuhl J, Leith J, Medoff DR, Fang L, Dickerson FB, Brown CH, et al. A comparison of adherence to hypoglycemic medications between type 2 diabetes patients with and without serious mental illness. Psychiatry Res 2011;188(1):109-14.

30. Kreyenbuhl J, Dixon LB, McCarthy JF, Soliman S, Ignacio RV, Valenstein M. Does adherence to medications for type 2 diabetes differ between individuals with vs without schizophrenia? Schizophr Bull 2010;36(2):428-35. https://doi.org/10.1093/schbul/sbn106

31. Gorczynski P, Firth J, Stubbs B, Rosenbaum S, Vancampfort D. Are people with schizophrenia adherent to diabetes medication? A comparative meta-analysis. Psychiatry Res 2017;250:17-24.

32. Young JQ, Kline-Simon AH, Mordecai DJ, Weisner C. Prevalence of behavioral health disorders and associated chronic disease burden in a commercially insured health system: findings of a case-control study. Gen Hosp Psychiatry 2015;37(2):101-8.Publisher's Note: Springer Nature remains neutral with regard to jurisdictional claims in published maps and institutional affiliations.

Publisher's Note Springer Nature remains neutral with regard to jurisdictional claims in published maps and institutional affiliations. 